International Mathematical Forum, 2, 2007, no. 16, 765 - 770

\title{
Ideal Amenability of Second Duals of Banach Algebras
}

\author{
M. Eshaghi Gordji ${ }^{(1)}$, F. Habibian ${ }^{(2)}$ and B. Hayati(i) \\ (1) Department of Mathematics, Faculty of Sciences, Semnan \\ University, Semnan, Iran \\ (2) Department of Mathematics, Isfahan University, Isfahan, Iran \\ (3) Department of Mathematics, Shahid Beheshti University, \\ Tehran, Iran \\ eimail:madjideg@walla.com \& fhabibian@ui.ac.ir \& \\ bahmanhayati@yahoo.com
}

\begin{abstract}
In this paper we study the ideal amenability of second duals of Banach algebras. We investigate relations between ideal amenability of the second dual of a Banach algebra with the first and the second Arens products.
\end{abstract}

Mathematics Subject Classification: 46H25

Keywords: Ideally amenable, Banach algebra, Derivation

\section{Introduction}

Let $\mathcal{A}$ be a Banach algebra and let $\mathcal{A}^{* *}$ be the second dual algebra of $\mathcal{A}$ endowed with the first or the second Arens product. Throughout this paper, the first and the second Arens product are respectively denoted by $\square$ and $\diamond$. These products can be defined by

$$
F \square G=w^{*} \lim _{i} \lim _{j} \hat{f}_{i} \hat{g}_{j} \quad \text { and } \quad F \triangleright G=w^{*} \lim _{j} \lim _{i} \hat{f}_{i} \hat{g}_{j}
$$

where $\left(f_{i}\right)$ and $\left(g_{j}\right)$ are nets of elements of $\mathcal{A}$ such that $f_{i} \longrightarrow F$ and $g_{j} \longrightarrow G$ in $w^{*}$-topology (see $[\mathrm{A}]$ and $\left.[\mathrm{D}-\mathrm{H}]\right)$. If $X$ is a Banach $\mathcal{A}$-bimodule, then a derivation from $\mathcal{A}$ into $X$ is a linear map $D$ such that for every $a, b \in \mathcal{A}$, $D(a b)=D(a) \cdot b+a \cdot D(b)$. If $x \in X$ and we define $\delta_{x}: \mathcal{A} \longrightarrow X$ by $\delta_{x}(a)=$ 
$a . x-x . a \quad(a \in \mathcal{A})$, then $\delta_{x}$ is a derivation. Derivations of this form are called inner derivations. Let $\mathcal{A}$ be a Banach algebra and $X$ be a Banach $\mathcal{A}$-bimodule, then $X^{*}$ is a Banach $\mathcal{A}$-bimodule if for each $a \in \mathcal{A}$ and $x \in X$ and $x^{*} \in X^{*}$ we define

$$
\left\langle a x^{*}, x\right\rangle=\left\langle x^{*}, x a\right\rangle, \quad\left\langle x^{*} a, x\right\rangle=\left\langle x^{*}, a x\right\rangle .
$$

A Banach algebra $\mathcal{A}$ is amenable if every derivation from $\mathcal{A}$ into every dual $\mathcal{A}$-bimodule is inner, equivalently if $H^{1}\left(\mathcal{A}, X^{*}\right)=\{0\}$ for every Banach $\mathcal{A}$ bimodule $X$, where $H^{1}\left(\mathcal{A}, X^{*}\right)$ is the first cohomology group of $\mathcal{A}$ with coefficients in $X^{*}[\mathrm{~J} 1],[\mathrm{R}],[\mathrm{H}]$.

A Banach algebra $\mathcal{A}$ is weakly amenable if $H^{1}\left(\mathcal{A}, \mathcal{A}^{*}\right)=\{0\}$ (the special case of $X=\mathcal{A})$ [B-C-D], [J2]. A Banach algebra $\mathcal{A}$ is ideally amenable if $H^{1}\left(\mathcal{A}, I^{*}\right)=\{0\}$ for every closed two-sided ideal $I$ in $\mathcal{A}$. Eshaghi-Gordji and Yazdanpanah have introduced the concept of ideal amenability of Banach algebras in $[\mathrm{G}-\mathrm{Y}]$, (see $[\mathrm{E}-\mathrm{H}])$.

\section{Some examples}

In this section, let $\mathcal{A}$ be a Banach algebra, $X$ a Banach $\mathcal{A}$-bimodule and let $Y$ be a Banach $\mathcal{A}$-submodule of $X$. We give some examples to show that $H^{1}\left(\mathcal{A}, X^{*}\right)=\{0\}\left(H^{1}(\mathcal{A}, X)=\{0\}\right)$ is not sufficient condition for $H^{1}\left(\mathcal{A}, Y^{*}\right)=\{0\}\left(H^{1}(\mathcal{A}, Y)=\{0\}\right)$. So, there are some examples to show that weak amenability dose not imply ideal amenability.

Example 1. Let $\mathcal{A}$ be a non weakly amenable Banach algebra with bounded left(or right) approximate identity, such that $H^{2}\left(\mathcal{A}, \mathcal{A}^{*}\right)=\{0\}$. We know that

$$
\{0\} \longrightarrow \operatorname{ker} \Delta \stackrel{i}{\longrightarrow} \mathcal{A} \hat{\otimes} \mathcal{A} \stackrel{\Delta}{\longrightarrow} \mathcal{A} \longrightarrow\{0\}
$$

is an exact sequence of Banach $\mathcal{A}$-bimodules, and

$$
\{0\} \longrightarrow \mathcal{A}^{*} \longrightarrow(\mathcal{A} \hat{\otimes} \mathcal{A})^{*} \longrightarrow(\operatorname{ker} \Delta)^{*} \longrightarrow\{0\}
$$

is an admissible exact sequence. $\mathcal{A}^{*}$ is a Banach $\mathcal{A}$-submodule of $(\mathcal{A} \hat{\otimes} \mathcal{A})^{*}$ and by Theorem 2.4.6 of $[\mathrm{R}]$ we have $H^{1}\left(\mathcal{A},(\mathcal{A} \hat{\otimes} \mathcal{A})^{*}\right) \simeq H^{2}\left(\mathcal{A}, \mathcal{A}^{*}\right)=\{0\}$. Now, let $X=(\mathcal{A} \hat{\otimes} \mathcal{A})^{*}$ and $Y=\mathcal{A}^{*}$, then $H^{1}(\mathcal{A}, X)=\{0\}$ but $H^{1}(\mathcal{A}, Y) \neq\{0\}$.

Example 2. Let $G=P S(2, \mathbb{R}), \mathcal{A}=X=L^{1}(G)$ and let $Y$ be the augmentation ideal of $\mathcal{A}$. Then we have $H^{1}\left(\mathcal{A}, Y^{*}\right) \neq\{0\}[\mathrm{J}-\mathrm{W}]$. On the other hand $\mathcal{A}$ is weakly amenable and so $H^{1}\left(\mathcal{A}, X^{*}\right)=\{0\}$. $\mathcal{A}$ is also an example of a weakly amenable Banach algebra which is not ideally amenable. 
Example 3. Let $G=P S(2, \mathbb{R})$ and let $I$ be the augmentation ideal of $L^{1}(G)$. Suppose that $X=\mathcal{A}=I^{\#}$ and $Y=I$. Then $H^{1}\left(\mathcal{A}, X^{*}\right)=\{0\} \neq H^{1}\left(\mathcal{A}, Y^{*}\right)$ $[\mathrm{G}-\mathrm{Y}]$.

Example 4. Let $\mathcal{A}$ be a Banach algebra. Then $\mathcal{A} \times \mathcal{A}$ is a Banach algebra by the following product and norm:

$$
\begin{gathered}
(a, x)(b, y)=(a b, a y+x b) \quad(a, b, x, y \in \mathcal{A}), \\
\|(a, b)\|=\|a\|+\|b\| \quad(a, b \in \mathcal{A}) .
\end{gathered}
$$

Let $\mathcal{A}$ be a weakly amenable Banach algebra with a bounded approximate identity such that span $\{a b-b a: a, b \in \mathcal{A}\}$ is dense in $\mathcal{A}$. Then by Theorem 5.4 of $[\mathrm{Zh}], \mathcal{A} \times \mathcal{A}$ is weakly amenable. Now let $0 \neq \varphi \in \Omega_{\mathcal{A}}$, then $D: \mathcal{A} \times \mathcal{A} \longrightarrow$ $\{0\} \times \mathcal{A}^{*}$ defined by $D(a, x)=(0, \varphi(a) \varphi)$ is a derivation. Since

$$
\begin{aligned}
D((a, x)(b, y)) & =D(a b, a y+x t) \\
& =(0, \varphi(a y+x b) \varphi) \\
& =(0,(\varphi(a) \varphi(y)+\varphi(x) \varphi(t)) \varphi) \\
& =(0,(\varphi(x) \varphi) b)+(0,(a \varphi(y)) \varphi) \\
& =(0, \varphi(x) \varphi)(b, y)+(a, x)(0, \varphi(y) \varphi) \\
& =D(a, x)(b, y)+(a, x) D(b, y) .
\end{aligned}
$$

If $D=\delta_{(0, f)}$ for some $f \in \mathcal{A}^{*}$, then we have

$$
\begin{aligned}
(0, \varphi(x) \varphi) & =D(a, x) \\
& =(a, x)(0, f)-(0, f)(a, x) \\
& =(x f, a f)-(x f, f a) \\
& =(x f-f x, a f-f a) .
\end{aligned}
$$

It shows that $(0, \varphi(x) \varphi)=D(a, x)=D(0, x)=(0,0)$, which is a contradiction with $\varphi \neq 0$. Now let $B=X=\mathcal{A} \times \mathcal{A}$ and $Y=\{0\} \times \mathcal{A}$. We have $H^{1}\left(B, X^{*}\right)=$ $\{0\}$ and $H^{1}\left(B, Y^{*}\right) \neq\{0\}$.

\section{Ideal Amenability of Second dual of a Ba- nach algebra}

In [Gh-L-W] Ghahramani, Loy and Willis studied some implications of amenability and weak amenability of $\mathcal{A}^{* *}$. In this paper we study the ideal amenability of the second dual of a Banach algebra. 
Theorem 3.1. Let $\mathcal{A}$ be a commutative Banach algebra. Suppose that $\mathcal{A}^{* *}$ is ideally amenable. If one of the following assertions holds:

(i) $\mathcal{A}$ is a left ideal in $\mathcal{A}^{* *}$.

(ii) $\mathcal{A}$ is a dual algebra.

(iii) $\mathcal{A}$ is Arens regular and every derivation from $\mathcal{A}$ into its dual is weakly compact.

Then $\mathcal{A}$ is ideally amenable.

Proof. Let $\mathcal{A}^{* *}$ be ideally amenable, then it is weakly amenable. If one of the (i) , (ii) or (iii) holds, then by Theorem 3.2 of [Gh-L-W], Theorem 2.2 of $[\mathrm{Gh}-\mathrm{L}]$ and Corollary 7.5 of $[\mathrm{D}-\mathrm{G}-\mathrm{V}], \mathcal{A}$ is weakly amenable. Since $\mathcal{A}$ is commutative, then $\mathcal{A}$ is ideally amenable $[\mathrm{G}-\mathrm{Y}]$.

Theorem 3.2. Let $\mathcal{A}$ be a Banach algebra. Consider $\mathcal{A}^{* *}$ endowed with the first Arens product. If $\mathcal{A}$ is an ideal of $\mathcal{A}^{* *}$ with a bounded approximate identity, then ideal amenability of $\mathcal{A}^{* *}$ implies ideal amenability of $\mathcal{A}$.

Proof. Let $I$ be a closed two sided ideal of $\mathcal{A}$. Then $I$ is an ideal in $\mathcal{A}^{* *}$. Now let $D: \mathcal{A} \longrightarrow I^{*}$ be a derivation, then there is a bounded derivation $\bar{D}: \mathcal{A}^{* *} \longrightarrow I^{*}$ which is an extension of $D[\mathrm{R}]$. Since $\mathcal{A}^{* *}$ is ideally amenable, then there exists $f \in I^{*}$ such that $\bar{D}=\delta_{f}$. Thus $D=\delta_{f}$ and $\mathcal{A}$ is ideally amenable.

For a Banach algebra $\mathcal{A}$, let $\mathcal{A}^{\text {op }}$ be the Banach algebra with underlying Banach space same as $\mathcal{A}$ and with product $\circ$ given by $a \circ b=b a$.

Lemma 3.3. Let $\mathcal{A}$ be a Banach algebra. Then $\mathcal{A}$ is ideally amenable if and only if $\mathcal{A}^{o p}$ is ideally amenable.

Proof. It is clear that $I$ is an ideal of $\mathcal{A}$ if and only if $I$ is an ideal of $\mathcal{A}^{o p}$. Now let $\mathcal{A}$ be ideally amenable and let $D: \mathcal{A}^{o p} \longrightarrow I^{*}$ be a continuous derivation, where $I$ is a closed ideal of $\mathcal{A}^{o p}$. Since for every $a, b \in \mathcal{A}, D(a b)=D(b \circ a)$ and $I$ is a closed ideal of $\mathcal{A}$, then $D: \mathcal{A} \longrightarrow I^{*}$ is a continuous derivation. But $\mathcal{A}$ is ideally amenable, so there is $\xi \in I^{*}$ such that $D(a)=a . \xi-\xi . a=-(a \circ \xi-\xi \circ a)$. This means that $\mathcal{A}^{o p}$ is ideally amenable. The converse is similar.

Lemma 3.4. Let $\mathcal{A}, \mathcal{B}$ be Banach algebras and let $\theta: \mathcal{A} \longrightarrow \mathcal{B}$ be a continuous isomorphism. If $\mathcal{A}$ is ideally amenable, then $\mathcal{B}$ is ideally amenable. 
Proof. It is evident.

Theorem 3.5. Let $\mathcal{A}$ be a Banach algebra with a continuous anti-isomorphism $\varphi: \mathcal{A} \longrightarrow \mathcal{A}$. Then $\left(\mathcal{A}^{* *}, \square\right)$ is ideally amenable if and only if $\left(\mathcal{A}^{* *}, \diamond\right)$ is ideally amenable.

Proof. Let $\left(\mathcal{A}^{* *}, \square\right)$ be ideally amenable and let $\varphi: \mathcal{A} \longrightarrow \mathcal{A}$ be a continuous anti-isomorphism. Take $F, G \in \mathcal{A}$ and let $\left(f_{i}\right),\left(g_{j}\right)$ be nets in $\mathcal{A}$ such that

$$
\text { weak }^{*}-\lim _{i} \hat{f}_{i}=F \quad \text { and } \quad w^{*}-\lim _{j} \hat{g}_{j}=G .
$$

Let $\varphi^{* *}:\left(\mathcal{A}^{* *}, \square\right) \longrightarrow\left(\mathcal{A}^{* *}, \diamond\right)$ be the second adjoint of $\varphi$. We have

$$
\begin{aligned}
\varphi^{* *}(F \square G) & =w^{*}-\lim _{i} w^{*}-\lim _{j} \varphi^{* *}\left(\hat{f}_{i} \hat{g}_{j}\right) \\
& =w^{*}-\lim _{i} w^{*}-\lim _{j}\left(\varphi\left(\widehat{\left(f_{i}\right) \varphi\left(g_{j}\right.}\right)\right) \\
& =w^{*}-\lim _{i} w^{*}-\lim _{j} \varphi^{* *}\left(\hat{g}_{j}\right) \varphi^{* *}\left(\hat{f}_{i}\right) \\
& =\varphi^{* *}(G) \diamond \varphi^{* *}(F) .
\end{aligned}
$$

Then $\varphi^{* *}$ is a continuous isomorphism from $\left(\mathcal{A}^{* *}, \square\right)$ onto $\left(\mathcal{A}^{* *}, \diamond\right)^{o p}$ and so by lemma $3.4,\left(\mathcal{A}^{* *}, \diamond\right)^{o p}$ is ideally amenable. Now, by lemma $3.3,\left(\mathcal{A}^{* *}, \diamond\right)$ is ideally amenable. The converse is similar.

Corollary 3.6. Let $\mathcal{A}$ be a commutative Banach algebra. Then $\left(\mathcal{A}^{* *}, \square\right)$ is ideally amenable if and only if $\left(\mathcal{A}^{* *}, \diamond\right)$ is ideally amenable.

Proof. If $\mathcal{A}$ is a commutative Banach algebra then the identity map is a continuous anti-isomorphism on $\mathcal{A}$. Now apply theorem 3.5.

Acknowledgement. The second author would like to thank the University of Isfahan for its financial support.

\section{References}

[A] R. Arens, The adjoint of a bilinear operation, Proc. Amer. Math. Soc. 2(1951), 839-848.

[B-C-D] W. G. Bade, P. G. Curtis and H. G. Dales, Amenability and weak amenability for Beurling and Lipschits algebra, Proc. London Math. Soc. (3) 55 (1987), 359-377. 
[D] H.G.Dales, Banach algebras and automatic continuity, Oxford, New York, 2000 .

[D-R-V] H. G. Dales, A.Rodriguez-Palacios and M. V. Velasco, The second transpose of a derivation, J. London Math. Soc. (2) 64 (2001) 707-721.

[D-H] J. Duncan and S. A. Hosseiniun, The second dual of Banach algebra, Proc. Roy. Soc. Edinburgh Set. A 84 (1979), 309-325.

[E-H] M. Eshaghi Gordji, S. A. R. Hosseiniun, Ideal Amenability of Banach Algebras on Locally Compact Groups, Proc. Indian Acad.Sci. Vol. 115 No.3, (2005), 319-325.

[G-Y] M. E. Gorgi, T. Yazdanpanah, Derivations into duals of ideals of $B a-$ nach algebras, proc. Indian Acad.Sci. Vol. 114 No.4, (2004), 399-403.

[Gh-L] F. Ghahramani, J. Laali, Amenability and topological centers of the second duals of Banach algebras, Bul. Austral. Math. soc. Vol. 65 (2002) 191-197.

[Gh-L-W] F. Ghahramani, R. J. Loy and G. A. Willis, Amenability and weak amenability of second conjugate Banach algebras, Proc. Amer. Math. Soc. Vol. 124, Num. 5 (1996).

[H] A. Ya. Helmeskii, The homology of Banach and topological algebras. Kluwer, (1989).

[J1] B. E. Johnson, Cohomology in Banach algebras, Mem. Amer. Math. Soc. 127 (1972).

[J2] —, Weak amenability of group algebras, Bull. Lodon Math. Soc. 23 (1991), 281-284.

[J-w] B. E. Johnson and M. C. White, A non-weakly amenable augmentation ideal, To appear .

[R] V. Runde, Lectures on amenability, Springer-Verlage Berlin Hedinberg New York,(2001).

[Zh] Y. Zhang, Weak amenability of a module extentions of Banach algebras, Trans. Amer. Math. soc. Vol354, Number 10, (2002) 4131-4151.

\section{Received: July 19, 2006}

